\title{
Zigarette weg, Bikini-Figur bleibt - meist
}

Wer mit dem Rauchen aufhört, wird dicker: Dieser Effekt schreckt viele ab, dabei nehmen die meisten kaum zu. Dafür profitiert das Herz in großem Maße.

Oft scheitert der Appell, mit dem Rauchen aufzuhören, weil viele Raucher Angst vor der Gewichtszunahme haben. Das kann bei jeder zweiten Frau und bei jedem vierten Mann der Fall sein, ergaben frühere Untersuchungen. Anhand von Daten der Framingham-Nachfolgestudie prüften Forscher um Dr. Carole Clair aus Boston die Hypothese, dass die Gewichtszunahme nach dem Rauchstopp den gesundheitlichen Nutzen dieser Maßnahme nicht senkt. In der prospektiven Kohortenstudie mit mehr als 3200 Teilnehmern über 25 Jahre wurde die Assoziation zwischen Gewichtszunahme über 4 Jahre nach Rauchstopp und die Inzidenz kardiovaskulärer Ereignisse analysiert, und zwar bei Erwachsenen mit und ohne Diabetes.

631 kardiovaskuläre Ereignisse (kvE) - KHK, zerebrovaskuläre Ereignisse, Herzinsuffizienz - und Claudicatio intermittens wurden dokumentiert. Nach Berücksichtigung des Alters und Geschlechts errechneten Clair et al. unter Nichtdiabetikern bei Rauchern eine kvE-Inzidenzrate von 5,9 (pro 100 Personen u. Untersuchungen), bei Exrauchern (Rauchstopp in den letzten 4 J.) von 3,2 und bei langjährigen Exrauchern (Rauchstopp vor $>4$ J.) eine Rate von 3,1. Bei den Nie-Rauchern lag die Rate bei 2,4.
Im Schnitt 1,4 Kilo mehr in vier Jahren

Im Mittel nahmen jene, die mit dem Rauchen aufgehört hatten, über den Zeitraum von vier Jahren 1,4 kg an Gewicht zu, also so viel wie Teilnehmer in mehreren anderen Studien. Allerdings nahmen $10-15 \%$ der Raucher beim Versuch, das Laster zu beenden, mehr als $10 \mathrm{~kg}$ zu.

Im Vergleich zu Rauchern war das kardiovaskuläre Risiko bei Teilnehmern, die mit dem Rauchen aufgehört hatten, signifikant verringert: Die Hazard Ratio (HR) lag bei Berücksichtigung des Alters und des Geschlechts bei jenen, die in den letzten vier Jahre aufgehört hatten, und bei jenen, die schon länger wieder Nichtraucher waren, bei 0,5. Das kardiovaskuläre Risiko war also um 50\% verringert. Bei Nierauchern war es aber im Vergleich zu Rauchern um 68\% verringert. Ähnliche Werte ergaben sich bei Diabetikern. Die Berücksichtigung von kardiovaskulären Risikofaktoren und Gewichtsveränderungen führte zu ähnlichen Ergebnissen.

Peter Leiner

Quelle: springermedizin.de basierend auf: Clair C et al. JAMA 2013; 309(10): 1014-21. 